\title{
Cluster magnetic fields through the study of polarized radio halos in the SKA era
}

\author{
Federica Govoni ${ }^{* 1 \dagger}$, Matteo Murgia ${ }^{1}$, Hao Xu${ }^{2}, \mathrm{Hui} \mathrm{Li}^{3}$, Michael Norman ${ }^{2}$, Luigina \\ Feretti $^{4}$, Gabriele Giovannini ${ }^{4,5}$, Valentina Vacca ${ }^{6}$, Gianni Bernardi ${ }^{7,8}$, Annalisa \\ Bonafede $^{9}$, Gianfranco Brunetti ${ }^{4}$, Ettore Carretti ${ }^{10}$, Sergio Colafrancesco ${ }^{11}$, Julius \\ Donnert $^{4}$, Chiara Ferrari ${ }^{12}$, Myriam Gitti ${ }^{4,5}$, Luigi lapichino ${ }^{13,14}$, Melanie \\ Johnston-Hollitt' ${ }^{15}$, Roberto Pizzo ${ }^{16}$, and Lawrence Rudnick ${ }^{17}$ \\ ${ }^{1}$ INAF - Osservatorio Astronomico di Cagliari, Italy; ${ }^{2}$ University of California at San Diego, \\ CA, USA; ${ }^{3}$ LANL Los Alamos, NM, USA; ${ }^{4}$ INAF - Istituto di Radioastronomia Bologna, Italy; \\ ${ }^{5}$ Dipartimento di Fisica e Astronomia, Universitá degli Studi di Bologna,Italy; ${ }^{6}$ MPA, Garching, \\ Germany; ${ }^{7}$ SKA SA, Cape Town, South Africa; ${ }^{8}$ Department of Physics and Electronics, Rhodes \\ University, Grahamstown, South Africa; ${ }^{9}$ University of Hamburg, Germany; ${ }^{10}$ CSIRO, \\ Australia; ${ }^{11}$ University of the Witwatersrand, SA; ${ }^{12}$ Observatoire de Nice, France; \\ ${ }^{13}$ Leibniz-Rechenzentrum, Garching, Germany; ${ }^{14}$ Universität Heidelberg, ZAH/ITA, Germany \\ ${ }^{15}$ Victoria University of Wellington, NZ; ${ }^{16}$ ASTRON, The Netherland; ${ }^{17}$ Minnesota Institute for \\ Astrophysics, University of Minnesota, USA \\ E-mail: fgovoni at oa-cagliari.inaf.it
}

\begin{abstract}
Galaxy clusters are unique laboratories to investigate turbulent fluid motions and large scale magnetic fields. Synchrotron radio halos at the center of merging galaxy clusters provide the most spectacular and direct evidence of the presence of relativistic particles and magnetic fields associated with the intracluster medium. The study of polarized emission from radio halos is extremely important to constrain the properties of intracluster magnetic fields and the physics of the acceleration and transport of the relativistic particles. However, detecting this polarized signal is a very hard task with the current radio facilities. We use cosmological magneto-hydrodynamical simulations to predict the expected polarized surface brightness of radio halos at $1.4 \mathrm{GHz}$. We compare these expectations with the sensitivity and the resolution reachable with the SKA1. This allows us to evaluate the potential for studying intracluster magnetic fields in the surveys planned for SKA1.
\end{abstract}

Advancing Astrophysics with the Square Kilometre Array

June 8-13, 2014

Giardini Naxos, Italy

\footnotetext{
*Speaker.

${ }^{\dagger}$ On behalf of the SKA Cosmic Magnetism Working Group
} 


\section{Introduction}

An unambiguous proof of the existence of $\mu \mathrm{G}$ magnetic fields spread in the intracluster medium is confirmed by radio observations (Carilli \& Taylor 2002, Govoni \& Feretti 2004). Information on intracluster magnetic fields can be obtained, in conjunction with X-ray observations of the hot gas, through the analysis of the rotation measure (RM) of radio galaxies lying in the background or embedded within the magnetized intracluster medium (Bonafede et al. 2015). Dedicated software tools and approaches based on a Fourier domain formulation have been developed to constrain the magnetic field power spectrum parameters on the basis of the rotation measure images (Enßlin \& Vogt 2003, Murgia et al., 2004, Laing et al. 2008, Kuchar \& Enßlin 2011). Typically, rotation measure images of cluster radio galaxies permit investigating the fluctuations of the intracluster magnetic field below a spatial scale of about 50-100 kpc. This does not, however, reach the Mpc scales of radio halos (Ferrari et al.2015, Cassano et al. 2015), which are large-scale diffuse synchrotron sources, located at the center of merging galaxy clusters. They have no optical counterpart and no obvious connection to cluster galaxies, and are therefore associated with the intracluster medium (Feretti et al. 2012).

The study of intracluster magnetic fields is one of the key science themes for the SKA Cosmic Magnetism program. The capability of SKA1 to perform spectro-polarimetric observations with high polarization purity, angular resolution, and sensitivity will permit us to dramatically improve our knowledge of the incidence, strength, and morphology of large-scale magnetic fields in the Universe. An All-Sky Polarization Survey at $\simeq 1 \mathrm{GHz}$ (Johnston-Hollitt et al. 2015), will have the potential of measuring the RM toward a large number of sources (Krause et al. 2009, Bogdanović et al. 2011) permitting derivation of a detailed description of the strength, structure, and radial decrease of cluster magnetic fields. At the same time, this survey will give the opportunity to investigate the total intensity and polarized emission of radio halos at an unprecedent sensitivity and resolution, permitting a detailed investigation of the magnetic field power spectrum in galaxy clusters. The possibility of obtaining both rotation measure and polarized halo emission in the same observation is fundamental to accurately determining the magnetic field spectrum over a large range of spatial scales (Govoni et al. 2006).

Cosmological simulations have been playing an important part in studying the intracluster magnetic field evolution of galaxy clusters (e.g. Dolag et al. 2002, Brüggen et al. 2005, Dubois \& Teyssier 2008, Ryu et al. 2008, Donnert et al. 2009, Xu et al. 2009, Dolag \& Stasyszyn 2009, Bonafede et al. 2011, Iapichino \& Brüggen 2012). Although the existence of cluster-wide magnetic fields is now well-established, their origin, which is ultimately important for understanding the evolution of the intracluster medium during the course of cluster formation, is still unclear (Widrow 2002). Magneto-hydrodynamical simulations of cluster formation have been performed with different initial magnetic fields; these have included random or uniform fields originating at high redshifts, or from the outflows of normal galaxies, or from active galaxies. The cluster magnetic fields of all these simulations are roughly in agreement at low redshifts. They predict $\mu \mathrm{G}$ level magnetic field strengths in the cluster centers and a decrease of the magnetic field strength with radius in the outer regions, in agreement with the observations. Cosmological magneto-hydrodynamical simulations are required at this stage of the project to explore the potential of the SKA1 for investigating intracluster magnetic fields, especially the possibility of detecting polarized radio halo emission. 


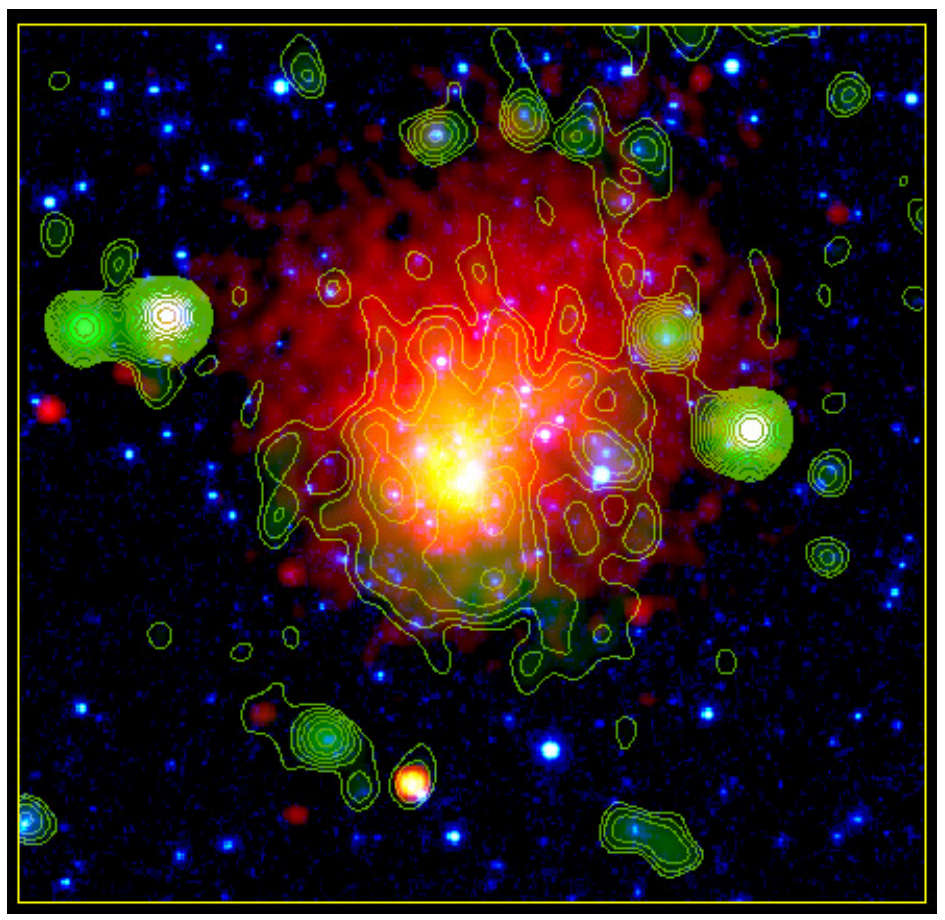

Figure 1: Galaxy cluster A665 at different wavelengths: optical (blue), X-ray (red), and radio (green contours). The radio image, showing an extended diffuse radio halo at the cluster center, has been obtained with the VLA at $1.4 \mathrm{GHz}$ (Vacca et al. 2010).

This chapter will introduce such simulations and their applications.

\section{Radio halo studies to investigate cluster magnetic fields}

Sensitive radio observations have revealed diffuse emission from the central regions of some merging galaxy clusters. These radio sources, extending over volumes of $\sim 1 \mathrm{Mpc}^{3}$ are called radio halos. They are diffuse, low-surface-brightness $\left(\simeq 1 \mu \mathrm{Jy} \mathrm{arcsec}^{-2}\right.$ at $\left.1.4 \mathrm{GHz}\right)$ and steep-spectrum ${ }^{1}$ $(\alpha>1)$ synchrotron sources with no obvious optical counterparts.

The brightness fluctuations of radio halos are closely related to the intracluster magnetic field structure and to the spatial distribution of the emitting relativistic particles (Tribble 1991). Therefore, information on the cluster magnetic fields can be derived from detailed radio halo images. Vacca et al. (2010) presented a study of the magnetic field power spectrum in the galaxy cluster A665, which contains a Mpc-scale radio halo (see Fig. 1). Under the assumption that the magnetic field energy density is in equipartition with that of the relativistic electrons and that these electrons have a power-law energy distribution, their modeling has proven successful in reproducing the observed total intensity fluctuations. The magnetic field model that best reproduces the observations in A665 is characterized by a central strength $\mathrm{B}_{0}=1.3 \mu \mathrm{G}$, with a magnetic energy density decreasing in proportion to the thermal gas density determined from the intracluster X-ray emission.

\footnotetext{
${ }^{1} S(v) \propto v^{-\alpha}$ structures, with $\alpha=$ spectral index.
} 

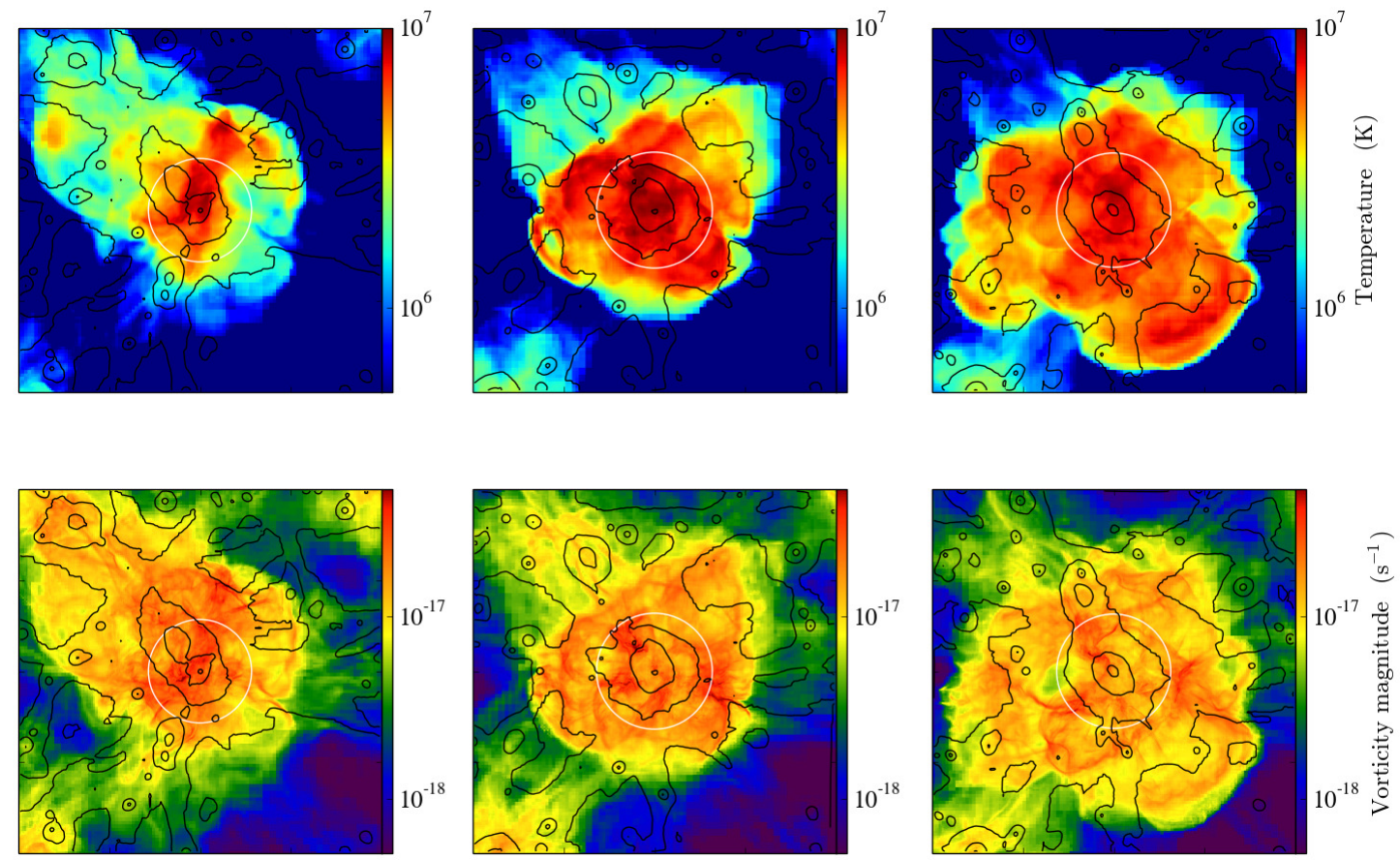

Figure 2: Series of projections (20 Mpc on a side, depth of $20 \mathrm{Mpc}$ ) showing gas temperature (upper row) and vorticity magnitude (magnitude of the velocity curl, $|\nabla \times \mathbf{v}|$, lower row) during a cluster major merger (Iapichino 2014). Three different times are shown, namely pre-merger phase (left, $z=0.43$ ), post-merger phase (center, $z=0.15$ ) and later phase (right, $z=0$ ). The region within one virial radius from the cluster centre is enclosed in the white circle. The plots are centered on a massive cluster $\left(M=1.3 \times 10^{15} M_{\odot}, R_{v i r}=\right.$ $3.1 \mathrm{Mpc}$ at $z=0)$.

Assuming a Kolmogorov magnetic field power spectrum, the outer scale of the magnetic field is $\Lambda_{\max } \sim 450 \mathrm{kpc}$.

Interesting morphological similarities between radio and X-ray images have been found in a number of clusters hosting a radio halo (e.g. Govoni et al. 2004). This similarity is generally valid for giant and regular halos. However, more irregular and asymmetric halos have been found in the literature. In these halos, the radio emission may show significant displacement from the X-ray emission. We investigated the statistics of the offset between the radio halo and the X-ray peak of the cluster emission. We found that halos can be quite asymmetric with respect to the X-ray gas distribution, and this becomes more relevant when halos of smaller size are considered (Feretti et al. 2012, Govoni et al. 2012). A possible explanation for this behavior can be attributed to the cluster magnetic field power spectrum. Indeed, on the basis of their magnetic field modeling, Vacca et al. (2010) found that if the outer scale of the magnetic field fluctuations is close to the observing beam, the halo appears smooth and rounded. Increasing the magnetic field correlation length results in a heavily distorted radio halo morphology and in a significant offset of the radio halo peak from the cluster center. Cluster merger events are expected to release a significant amount of energy into the intracluster medium. This energy is injected on large spatial scales, and then turbulent cascades may transport energy to smaller scales. This process is expected to affect the micro-physics of the 
intracluster medium : particle transport and acceleration, heating of the intracluster medium, and magnetic field amplification (e.g. Roettiger et al. 1999, Ricker \& Sarazin 2001, Dolag et al. 2005, Vazza et al. 2009, Vazza et al. 2011, Xu et al. 2010, Xu et al. 2011, Donnert et al. 2013, Brunetti \& Jones 2014). We thus expect a different morphology in the radio halo structure between young and old mergers. Young and smaller systems should have a magnetic field correlation length larger than in the more extended, and dynamical older, radio halos (Govoni et al. 2012). Initially the magnetic field is indeed stirred by the gas-dynamics on scales that are larger than that in dynamically older systems. In addition, both particle acceleration and diffusion/transport depend on turbulent properties (Brunetti \& Jones 2014) and a smoother spatial distribution of particles is generally expected in the case of old radio halos. In this framework of energy cascades, smaller halos may have a more distorted morphology if they are young systems in which the energy is still on large scales. They would have a magnetic field correlation length larger than in the more extended, and dynamical older, radio halos. This could be tested by comparing SKA1 observations with the dynamical state of the clusters, as seen in the optical and X-ray bands.

The turbulent dynamo is a widely accepted mechanism for amplifying the magnetic field in the intracluster medium to values well above the level predicted by adiabatic compression. This process is likely to also be important in the cluster outskirts (around and beyond $0.5 R_{v i r}$ ), where simulations predict a pressure support by turbulent motions of the order of 10-30 per cent of the total pressure on scales of a few times $100 \mathrm{kpc}$ (Vazza et al. 2011). Interestingly, recent hydrodynamical simulations (Iapichino 2014, Miniati 2014) show that a major merger event can stir the gas and drive turbulent flow even beyond the cluster virial radius. This could persist up to a few Gyr after the merger, thus providing an important mechanism for amplifying magnetic fields not only in cluster cores, but also in the outer regions (see Fig. 2).

\subsection{Polarized intensity of radio halos}

The detection of polarized emission from radio halos would be extremely important to investigate the magnetic field power spectrum in galaxy clusters and to find merger shocks in the intracluster medium not visible in the X-ray images. Murgia et al. (2004) simulated 3D magnetic fields in galaxy clusters with a single power-law power spectrum of the magnetic field fluctuations $\left|B_{k}\right|^{2} \propto k^{-n}$. They investigated how different magnetic field power spectra affect the shape and the polarization properties of radio halos. Models with $n>3$ and $\Lambda_{\max }>100 \mathrm{kpc}$ result in magnetic fields whose energy is larger on the large spatial scales, thus giving rise to possible filamentary and polarized radio halos. Models with $n<2$, instead, having most of the magnetic field energy on small spatial scales, will give rise to halos with a more regular morphology, and very little polarization.

Typically, radio halos are found to be unpolarized when observed with current radio facilities. But, in agreement with the expectations of Murgia et al. (2004), filaments of polarized emission associated with a radio halo have been detected (see Fig. 3) in A2255 (Govoni et al. 2005) and MACS J0717.5+3745 (Bonafede et al. 2009). The radio halo in MACS J0717+3745 is one of the most powerful radio halos observed so far, with a mean fractional polarization of $\simeq 5 \%$ at $1.4 \mathrm{GHz}$. The detection of polarized emission indicates that the magnetic field power spectrum in the cluster should be steeper than $n=3$, and that its maximum scale $\Lambda_{\max }$ should be $>100 \mathrm{kpc}$. On the basis of the interpretation by Govoni et al. (2005), in A2255 a single power law cannot account for the 

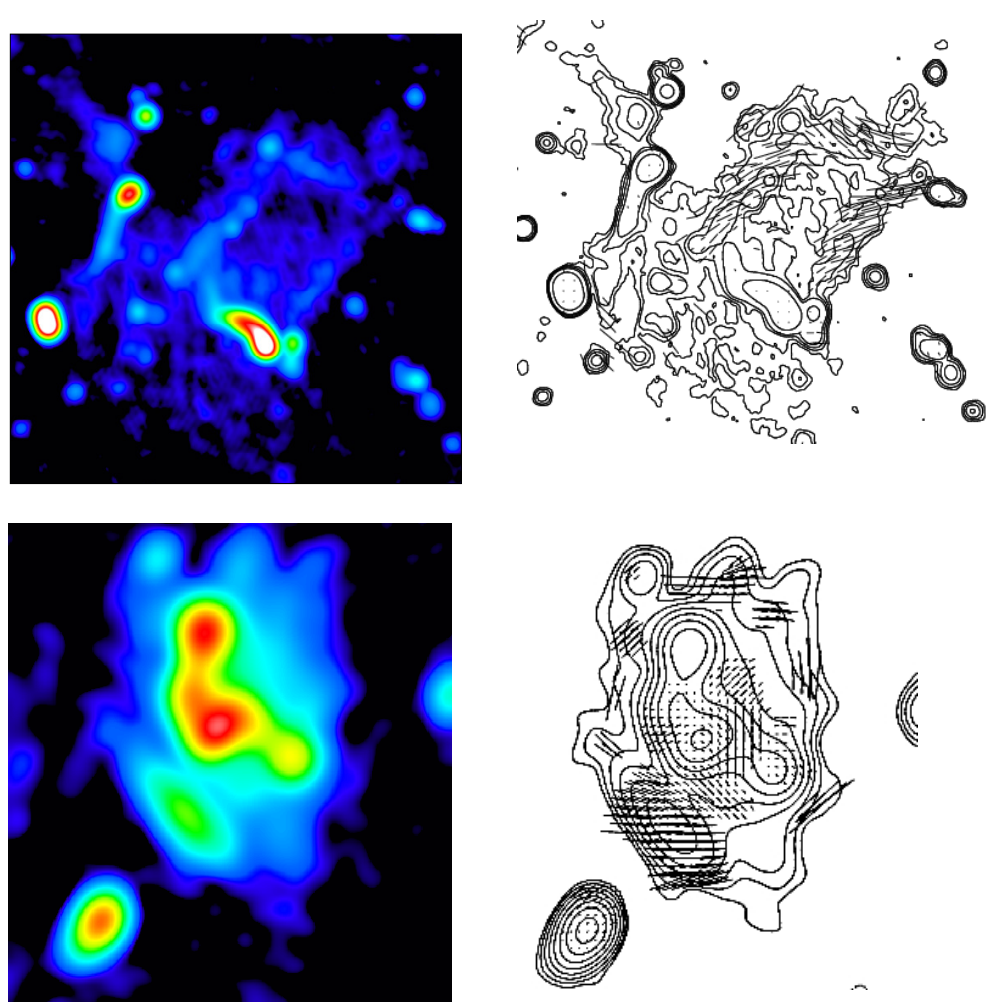

Figure 3: Total intensity and polarization vectors of the radio halos in which a polarized signal has been detected up to now. Top: A2255 (Govoni et al. 2005). Bottom: MACS J0717.5+3745 (Bonafede et al. 2009).

observed polarization. A power spectrum with spectral index $n=2$ at the cluster center and $n=4$ at the cluster periphery is needed to produce the observed polarized emission. To explain the highly polarized structures observed in the radio halo it is necessary that most of the magnetic field energy density is on large scales at the cluster periphery. Pizzo et al. (2011) confirmed that the polarized filaments are located at the outskirts of the cluster, but in their interpretation such polarization is not associated with the radio halo emission.

There are several reasons why halo polarization is usually not detected. The orientation of the magnetic field likely changes many times during the path through the cluster. Faraday rotation along the line of sight will suppress the net polarization, due to multiple field reversals along each path. Further depolarization is likely within each beam (beam-depolarization) if the beam size is larger than the angular scale of coherent magnetic field regions.

Recently, Govoni et al. (2013) using cosmological magneto-hydrodynamical simulations showed that radio halos are intrinsically polarized at full-resolution. The fractional polarization at the cluster center is $\simeq 15-35 \%$ with values varying from cluster to cluster and increasing with the distance from the cluster center. However, the polarized signal is undetectable if observed with the comparatively shallow sensitivity and low resolution of current radio interferometers.

A high level of intrinsic polarization is also qualitatively in agreement with the early postmerger phase in major mergers, when merger shocks are launched in the cluster's innermost region. 


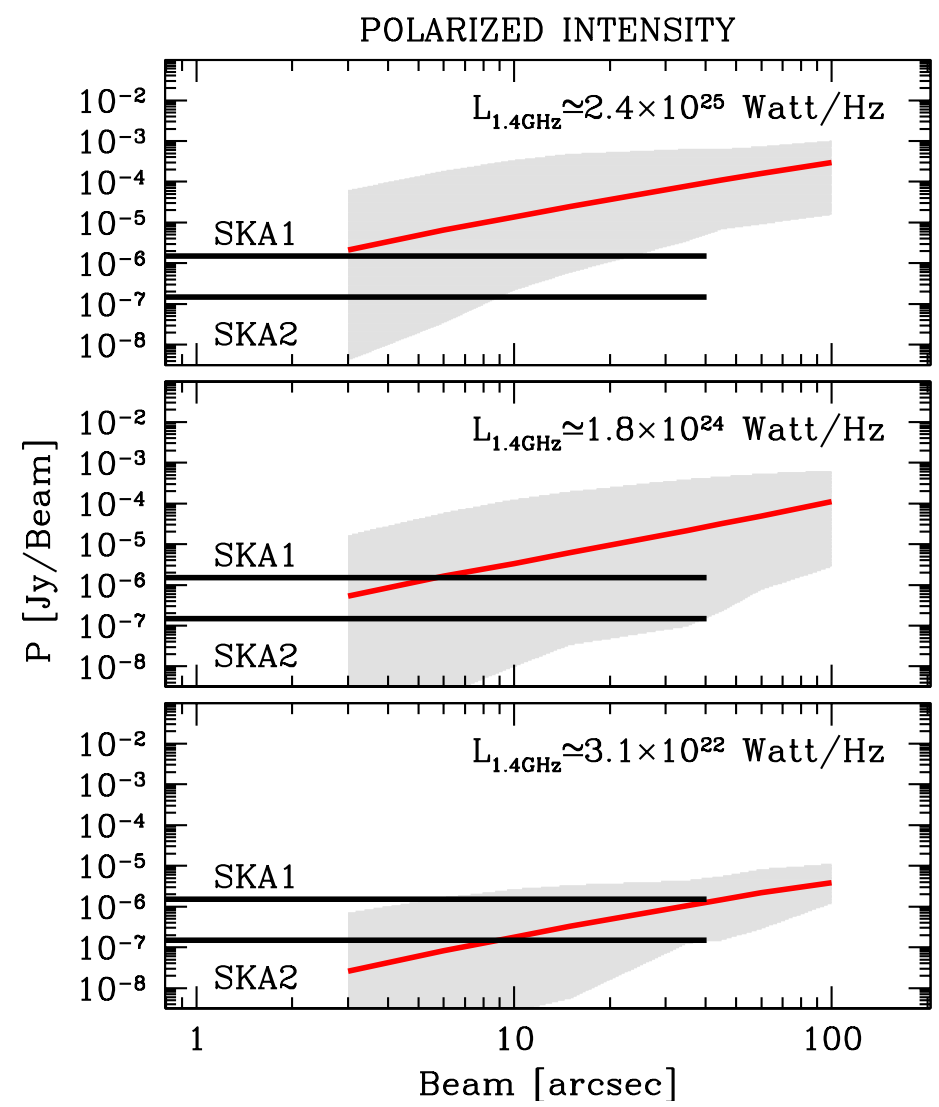

Figure 4: Polarized intensity surface brightness at $1.4 \mathrm{GHz}$ as a function of the beam size $\left(3^{\prime \prime}-100^{\prime \prime}\right)$ for three mock radio halos of different luminosities (Govoni et al. 2013), obtained by using cosmological magnetohydrodynamical simulations by Xu et al. (2012). The solid red line shows the average brightness, while the shaded region shows the maximum and minimum brightness fluctuations. The simulated surface brightness is compared with a typical sensitivity expected for SKA1 $(\sigma \simeq 1 \mu \mathrm{Jy} /$ beam $)$ and SKA2 $(\sigma \simeq 0.1 \mu \mathrm{Jy} / \mathrm{beam})$. The sensitivity refers to the $3-\sigma$ limit.

Simulations of major mergers (e.g. Iapichino 2014, Miniati 2014), suggest that, in this early phase, the flow in the core is dominated by the shock propagation, rather than by turbulent stirring. Targeted studies are needed to determine the duration of this transition in comparison with the radio halo lifetime, and the resulting level of polarization.

\section{Magneto-hydrodynamical simulations}

In preparation for SKA, several next-generation radio telescopes and upgrades are being constructed around the world. Among them, APERTIF, ASKAP, LOFAR, Meerkat, and the Jansky VLA all offer the chance to explore the polarization properties of cluster diffuse emission. Deep polarization sky surveys are being planned for many of these telescopes. The WODAN survey (Westerbork Observations of the Deep APERTIF Northern-Sky; Röttgering et al., 2011) will use APERTIF to explore at $1.4 \mathrm{GHz}$ the northern sky. This survey will provide a spatial resolution of $\simeq 15^{\prime \prime}$ and a sensitivity of about $10 \mu \mathrm{Jy} / \mathrm{beam}$. A similar performance will be reached in the 
southern sky with ASKAP through the polarization survey POSSUM (POlarization Sky Survey of the Universe's Magnetism; Gaensler et al. 2010). These resolution and sensitivity values are very promising for detection of the polarized emission of the most powerful $\left(L_{1.4 \mathrm{GHz}} \simeq 2.4 \times 10^{25}\right.$ Watt $/ \mathrm{Hz})$ radio halos, while halos of intermediate luminosity $\left(L_{1.4 \mathrm{GHz}} \simeq 1.8 \times 10^{24} \mathrm{Wat} / \mathrm{Hz}\right)$ will still be hardly detectable (Govoni et al. 2013) at this phase.

The high sensitivity and large bandwidths provided by SKA1-MID and SKA1-SUR can be used to exploit the rotation measure synthesis technique and to extract polarized emission at different Faraday depths (Burn 1966, Brentjens \& de Bruyn 2005). Pizzo et al. (2011) showed that this technique is very effective in studying the polarized emission of both the discrete sources and the diffuse emission in a comprehensive way. However, alternative techniques like the Faraday synthesis (Bell \& Enßlin 2012), modeling of the fractional Q and $U$ as a function of $\lambda^{2}$ (Farnsworth et al. 2011), and stacking radio polarization (Stil \& Keller 2015) are also under investigation as ways to best use the capabilities of the SKA and its precursors. We analyzed (Govoni et al. 2013) how the polarization of radio halos will appear at $1.4 \mathrm{GHz}$, when observed with a bandwidth of $1 \mathrm{GHz}$. In Fig. 4, we present the expected polarized emission of three mock radio halos (characterized by different radio luminosities) as a function of the angular resolution, obtained by applying the rotation measure synthesis. We tentatively represent the $3-\sigma$ limit expected with SKA1 $(\sigma \simeq 1 \mu \mathrm{Jy} / \mathrm{beam})$ and SKA2 $(\sigma \simeq 0.1 \mu \mathrm{Jy} /$ beam $)$, by assuming that the confusion limit is negligible in polarization. Fig. 4 shows that at the sensitivity reachable with the SKA1 it will be possible to detect, at high-resolution $\left(\simeq 5-10^{\prime \prime}\right)$, polarized emission in radio halos of high and intermediate luminosity. For fainter halos, $\left(L_{1.4 G H z} \simeq 3.1 \times 10^{22} \mathrm{Watt} / \mathrm{Hz}\right)$, the detection of the polarized emission at high resolution is very difficult even for SKA2. Our simulations are based on equipartition between relativistic electrons and magnetic fields and assume that relativistic particles have a power law energy distribution. Simulations including models for acceleration/transport will further shed light on this topic.

\section{Conclusions}

The detection of polarized signal from radio halos is a very hard task with current radio facilities. Therefore, this is an interesting science case for SKA1. The detection of polarized emission from radio halos may be the key for investigating the magnetic field power spectrum in galaxy clusters and to find merger shocks in the intracluster medium not visible in X-ray images. Indeed, merger shocks with Mach numbers $M \simeq 1-2$, do not produce enough heating to be visible in $\mathrm{X}$-ray images. But, through amplification by compression, they may be detectable in polarization (even before they are seen in total intensity) when observed with SKA1. It is likely that thanks to SKA1 we will be able to detect the polarized emission in a large number of radio halos, obtaining a clearer picture of the power spectrum of the magnetic field fluctuations over the entire volume of the cluster. 


\section{REFERENCES}

Bell, M. R., \& Enßlin, T. A., 2012, A\&A, 540, A80

Bogdanović, T., Reynolds, C. S., Massey, R., 2011, ApJ, 731, 7

Bonafede, A., Feretti, L, Giovannini, G., et al., 2009, A\&A, 503, 707

Bonafede, A., Dolag, K., Stasyszyn, F., Murante, G., \& Borgani, S. 2011, MNRAS, 418, 2234

Bonafede, A., Vazza, F., Brüggen, M., et al., 2015, "Unravelling the origin of large-scale magnetic fields in galaxy clusters and beyond through Faraday Rotation Measures with the SKA" in proc. Advancing Astrophysics with the Square Kilometre Array, PoS(AASKA14)095

Brentjens, M.A., de Bruyn, A.G., 2005, A\&A, 441, 1217

Brüggen, M., Ruszkowski, M., Simionescu, A., Hoeft, M., Dalla Vecchia, C., 2005, ApJ, 631, L21

Brunetti, G., \& Jones, T.W., 2014, International Journal of Modern Physics D, 23, 30007

Burn, B.J., 1966, MNRAS, 133, 67

Carilli, C. L., \& Taylor, G. B. 2002, Annual Review of Astronomy and Astrophysics, 40, 319

Cassano, R., Bernardi, G., Brunetti, G., et al., 2015, "Cluster Radio Halos at the crossroads between astrophysics and cosmology in the SKA era" in proc. Advancing Astrophysics with the Square Kilometre Array, PoS(AASKA14)073

Dolag, K., Bartelmann, M., Lesch, H., 2002, A\&A, 387, 383

Dolag, K., Vazza, F., Brunetti, G., \& Tormen, G., 2005, MNRAS, 364, 753

Dolag, K., \& Stasyszyn, F. 2009, MNRAS, 398, 1678

Donnert, J., Dolag, K., Lesch, H., Müller, E. 2009, MNRAS, 392, 1008

Donnert, J., Dolag, K., Brunetti, G., Cassano, R., 2013, MNRAS, 429, 3564

Dubois, Y., \& Teyssier, R., 2008, A\&A, 482, L13

Enßlin T.A., \& Vogt C., 2003, A\&A, 401, 835

Farnsworth, D., Rudnick, L., Brown, S., 2011, AJ, 141, 191

Feretti, L., Giovannini, G., Govoni, F., \& Murgia, M. 2012, The Astronomy and Astrophysics Review, 20, 54

Ferrari, C., Dabbech, A., Smirnov, O., et al., 2015, "Non-thermal emission from galaxy clusters: feasibility study with SKA" in proc. Advancing Astrophysics with the Square Kilometre Array, PoS(AASKA14)075

Gaensler, B.M., Landecker, T.L., Taylor, A.R., \& POSSUM Collaboration, 2010, Bulletin of the American Astronomical Society, 42, \#470.13

Govoni, F., Markevitch, M., Vikhlinin, A., et al. 2004, ApJ, 605, 695

Govoni, F., \& Feretti, L. 2004, International Journal of Modern Physics D, 13, 1549

Govoni, F., Murgia, M., Feretti, L., et al., 2005, A\&A, 430, L5

Govoni, F., Murgia, M., Feretti, L., et al., 2006, A\&A, 460, 425

Govoni, F., Ferrari, C., Feretti, L., et al. 2012, A\&A, 545, A74

Govoni, F., Murgia, M., Xu, H., et al., 2013, A\&A, 554, A102

Iapichino, L., \& Brüggen, M. 2012, MNRAS, 423, 2781

Iapichino, 2014, private communication

Johnston-Hollitt, M., et al., 2015, "Using SKA Rotation Measures to Reveal the Mysteries of the Magnetised Universe" in proc. Advancing Astrophysics with the Square Kilometre Array, PoS(AASKA14)092 
Krause, M., Alexander, P., Bolton, R., et al., 2009, MNRAS, 400, 646

Kuchar, P., \& Enßlin, T.A., 2011, A\&A 529, A13

Laing, R.A., Bridle, A.H., Parma, P., Murgia, M., 2008, MNRAS, 391, 521

Miniati, F. 2014, ApJ, 782, 21

Murgia M., Govoni F., Feretti L., et al., 2004, A\&A, 424, 429

Pizzo, R.F., de Bruyn, A.G., Bernardi, G., Brentjens, M.A., 2011, A\&A, 525, A104

Ricker, P.M., \& Sarazin, C.L., 2001, ApJ, 561, 621

Roettiger, K., Stone, J.M., Burns, J.O., 1999, ApJ, 518, 594

Röttgering, H., Afonso, J., Barthel, P., et al., 2011, Journal of Astrophysics and Astronomy, 32, 557

Ryu, D., Kang, H., Cho, J., Das, S., 2008, Science, 320, 909

Stil, J.M., Keller, B.W., 2015, "Stacking for Cosmic Magnetism with SKA Surveys" in proc. Advancing Astrophysics with the Square Kilometre Array, PoS(AASKA14)112

Tribble, P.C., 1991, MNRAS, 253, 147

Vacca, V., Murgia, M., Govoni, F., et al., 2010, A\&A, 514, A71

Vazza, F., Brunetti, G., Kritsuk, A., et al., 2009, A\&A, 504, 33

Vazza, F., Brunetti, G., Gheller, C., Brunino, R., Brüggen, M., 2011, A\&A, 529, A17

Widrow, L. M. 2002, Reviews of Modern Physics, 74, 775

Xu, H., Li, H., Collins, D.C., Li, S., Norman, M.L., 2009, ApJ, 698, L14

Xu, H., Li, H., Collins, D.C., Li, S., Norman, M.L., 2010, ApJ, 725, 2152

Xu, H., Li, H., Collins, D.C., Li, S., Norman, M.L., 2011, ApJ, 739, 77

Xu, H., Govoni, F., Murgia, M., et al. 2012, ApJ, 759, 40 